\title{
Determinants of Response to Cardiac Resynchronization Therapy
}

\author{
JAGMEET SINGH, MD, DPHIL
}

Massachusetts General Hospital, Boston, MA

\begin{abstract}
Cardiac resynchronization therapy has been shown to improve the functional class and quality of life, reduce mitral regurgitation, and remodel the heart favourably, while reducing heart failure hospitalization and overall mortality in this large subgroup of heart failure (HF) patients.
\end{abstract}

KEYWORDS. Cardiac resynchronization therapy, heart failure, non-responsive.
ISSN 2156-3977 (print) ISSN 2156-3993 (online)

(C) 2016 Innovations in Cardiac Rhythm Management

\section{Introduction}

Cardiac resynchronization therapy (CRT) is an effective and established therapy for patients with medically refractory heart failure with left ventricular systolic dysfunction and a wide QRS complex. CRT has been shown to improve the functional class and quality of life, reduce mitral regurgitation, and remodel the heart favorably, while reducing heart failure hospitalization and overall mortality in this large subgroup of heart failure (HF) patients. Despite these beneficial effects of CRT, a significant minority (approximately 30\%) of patients remains non-responsive to this therapeutic modality. Notably, there are three broad determinants of resynchronization therapy response: firstly, selecting the "appropriate" patient, secondly, implanting the left ventricular lead in the correct location, and thirdly, optimally programming the devices.

\section{Patient selection}

Echocardiography-guided cardiovascular imaging to better define mechanical dyssynchrony as a patient selection strategy has had challenges, preventing it from becoming a mainstream strategy. ${ }^{1,2}$ Imaging approaches

\footnotetext{
Dr. Singh has received research grants from Boston Scientific, Sorin Group, Medtronic, and St. Jude Medical; is a member of the steering committee/DSMB Consulting for Boston Scientific, CardioInsight, Medtronic, St. Jude Medical, Sorin Group, BackBeat Inc., theheart. org, Respicardia Inc., and Impulse Dynamics; and has participated in educational symposia for Boston Scientific, Medtronic, St. Jude Medical, and Sorin Group.

Manuscript received February 29, 2016, Final version accepted April 17, 2016.

Address correspondence to: Jagmeet Singh, Associate Chief, Cardiology Division Massachusetts General Hospital Heart Center, Professor of Medicine, Harvard Medical School, 55 Fruit St. Boston, MA 02114. E-mail: jsingh@mgh.harvard.edu
}

such as magnetic resonance imaging (MRI) and computed tomography CT are evolving as tools, and their ability to provide information on cardiac function, along with the venous anatomy and scar location, may help us better select patients. At this time, we still rely on one of the most simplistic measures from the 12-lead electrocardiogram-the surface QRS signal. The QRS duration has gained the notoriety of being one of the most important criteria for identification of patients in whom ventricular asynchrony could be ameliorated by left ventricular pacing. ${ }^{3}$

When looking at the impact of QRS within randomized clinical trials, it is evident that the wider the QRS the better. Patients who have a wide QRS have more mechanical dyssynchrony, and a greater extent of mechanical dyssynchrony is often times more forgiving of a suboptimal left ventricular (LV) lead position. During CRT, the right ventricular (RV) and LV leads generate two ventricular wavefronts and the benefit of CRT depends on the effective fusion of these wavefronts, synchronizing the contraction of the ventricles. Given that this pattern of electrical activation sequence in left bundle branch block (LBBB), is associated with delayed mechanical activation of the posterior basal lateral wall, targeting this region for LV pacing has been the standard approach for conventional CRT implants. In contrast to LBBB, patients with a right bundle branch block (RBBB) or non-specific intraventricular conduction delay (IVCD) have differing activation patterns and may therefore not benefit as much from CRT. Of note, there is substantial heterogeneity of activation wave fronts within bundle branch type that may be further modified by substrate phenotype including myocardial scar and inflammation. Understanding the electrical pathophysiology and rationale for CRT frames the most recent North American guidelines for CRT therapy restricting a Class I recommendation only for patients with LBBB and QRS $\geq 150$ 
msec. Meta-analysis of several landmark trials (COMPANION, CARE-HF, REVERSE, MADIT-CRT, RAFT) suggested that significant reduction of composite clinical events was present only in patients with baseline QRS $\geq$ $150 \mathrm{msec}$. Data from a meta-analysis in 2010, significantly influenced the European Society of Cardiology (ESC) and, subsequently, the Heart Failure Society of America (HFSA) and Heart Rhythm Society (HRS), all of whom endorsed a QRS of $150 \mathrm{~ms}$ to ensure response among patients with non-LBBB. ${ }^{4}$ As alluded to above, patients with non-LBBB have poorer outcomes largely because of variability in the activation sequence of the heart, and the conventional implant procedure may not be as effective. ${ }^{5}$ Beyond QRS morphology and duration, there are several other baseline clinical features that may impact the response to CRT. In atrial fibrillation, there is loss of atrio-ventricular synchrony as well as suboptimal effective biventricular capture on account of irregular and high ventricular rates, leading to the suboptimal delivery of biventricular pacing. In a sub-analysis of patients with atrial fibrillation enrolled in the RAFT trial, it was observed that while CRT did reduce HF hospitalization (40\% risk reduction), it had no influence on cardiovascular death. In addition to QRS duration and atrial fibrillation, several other factors have been shown to influence the efficacy of CRT including medical comorbidities (chronic renal insufficiency), ${ }^{6}$ hemodynamic pathology (pre-capillary pulmonary hypertension), ${ }^{7}$ and abnormalities of LV substrate (non-revascularizable coronary artery disease, myocardial scar). ${ }^{8}$ There has also been a suggestion that women may benefit from CRT more so than men, particularly in patients with LBBB and even at QRS duration $<150 \mathrm{msec}^{9}$ Whether these factors only satisfy the efficacy of CRT versus obviate the benefit of biventricular pacing remain to be assessed in future prospective studies.

\section{LV lead placement}

Response to LV lead location involves multiple variables. Covariates that can impact response to LV lead location include the substrate, ischemic or non-ischemic status, presence or absence of scar, surrounding tissue health, electrical issues, such as RV-LV fusion, depolarization wavefront, proximity of the LV lead to the Purkinje network system, orientation of the electrode, or myofibrillar pattern (Figure 1). Multiple variables can impact how the patient responds to a particular LV lead location. Thus, even if the lead is placed in the correct location, the patient may not respond because these different variables can influence the impact of pacing.

The conventional approach to implantation of the left ventricular lead is via a transvenous approach targeting the lateral or posterolateral location within a branch of the coronary sinus veins (Figure 2). This strategy takes into consideration the expected pattern of delayed electrical and mechanical activation of the lateral and posterolateral wall, particularly in patients with LBBB. Amongst the biggest challenges to appropriately targeting the left ventricular lead is the heterogeneity of coronary sinus venous anatomy.

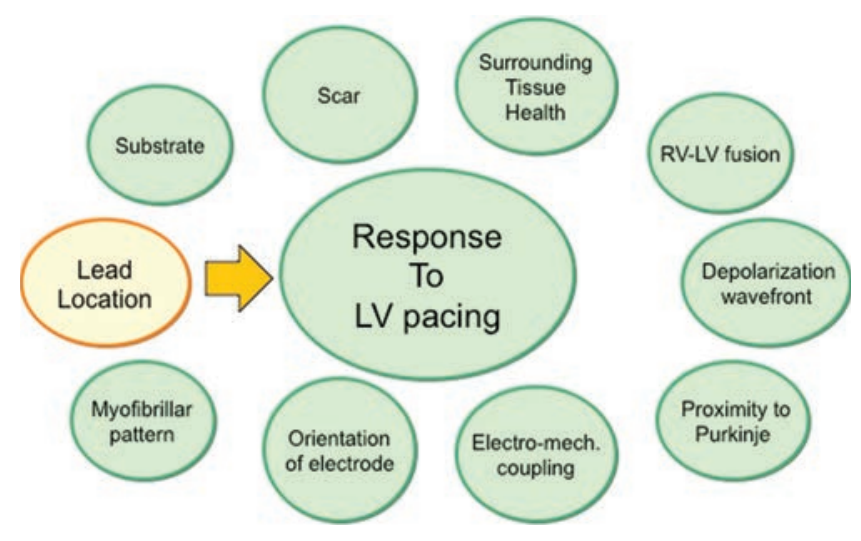

Figure 1: Left ventricular pacing location and response: thinking beyond anatomical targeting.

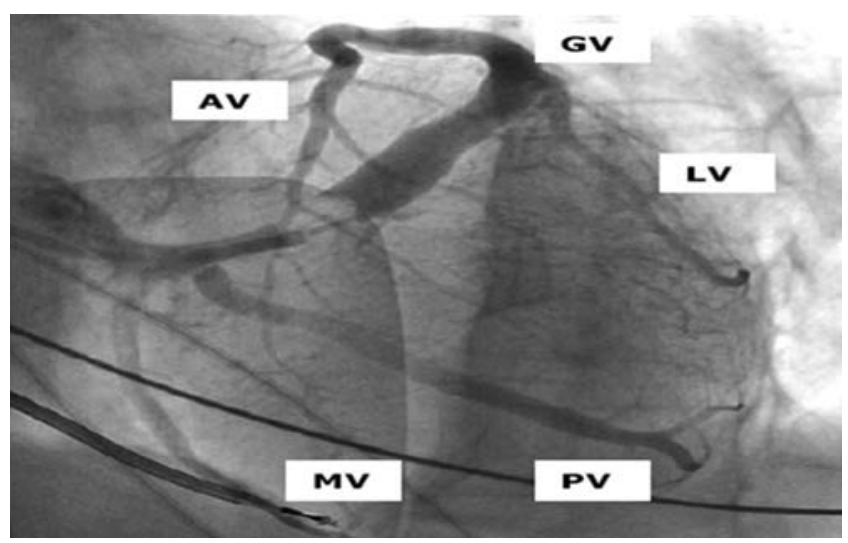

Figure 2: Suggested optimal locations are the postero-lateral wall and lateral wall. The suggested mechanics resets the electrical activation sequence and reverses free wall dyssynchrony. Reprinted from Journal of the American College of Cardiology, 46/1, Jagmeet P. Singh, MD, PhD, Stuart Houser, MD, E. Kevin Heist, MD, PhD, Jeremy N. Ruskin, MD, The Coronary Venous Anatomy: A Segmental Approach to Aid Cardiac Resynchronization Therapy, Pages 68-74, 2005, with permission from Elsevier.

Response to CRT is often variable even when the LV lead is placed in this optimal anatomic position, reflecting the complex interaction of myocardial substrate (e.g. scar), variability of the ventricular wavefront activation even within similar bundle branch morphologies, as well as RV pacing-induced shifts in left ventricular activation. Anatomically, placement of the LV lead can be organized along the long and short axes. Placement of the LV lead in an apical position has been shown to be associated with a worse clinical outcome. Compared to non-apical locations, the apical placement is usually neither electrically distant or adequately anatomically separated from the RV lead. Beyond anatomic targeting, individualized approaches include identification of regions of maximal electrical delay, maximal mechanical delay, or lead positions that will enhance the hemodynamics. Given that CRT is a 
form of electrical therapy for patients with abnormalities of electrical conduction, it remains innate that targeting the site of latest electrical activation may yield the most favorable CRT response. While the site of latest electrical delay has been identified using various methods including three-dimensional non-contact endocardial mapping, a more practical strategy has been the use of intracardiac electrograms (EGM) to measure the delay between the surface QRS and the initial sensed intracardiac signal of the LV lead (QLV). ${ }^{10}$ This difference can be further corrected for baseline QRS duration to yield the LV lead electrical delay (LVLED). ${ }^{11}$ Lead placement at sites of increasing QLV is associated with greater rates of $\mathrm{LV}$ reverse remodeling and improvement in patient symptoms. Recent efforts have also demonstrated the feasibility of newer electro-anatomical mapping catheters to define sites of electrical delay.

While mechanical dyssynchrony has historically failed to predict CRT response or identify patients with narrow QRS who may benefit from CRT, newer modalities of mechanical dyssynchrony assessment (e.g. speckle tracking and cardiac MRI) are being continually evaluated and refined. In the STARTER (Speckle Tracking Assisted Resynchronization Therapy for Electrode Region) trial, a lead targeting strategy at sites of latest activation with avoidance of areas of scar was associated with significant reduction in composite outcome. ${ }^{12}$ Of note, exact concordance was only achieved in $30 \%$ of patients highlighting the limitations of coronary venous anatomy. To target the area of mechanical dyssynchrony, it is important to distinguish dyssynchrony from the presence or absence of scar, because oftentimes the scar itself may mimic mechanical dyssynchrony. In prospective evaluations, Bose et al. and others, ${ }^{8}$ and de Roest et al. ${ }^{13}$ have found that the likelihood of response is low when the lead is placed over a segment of scar. When the lead is placed over an ischemic segment, the response is better but is still low compared with placing the lead over an area of normal viable myocardium. These results indicate the need to individualize the LV lead implant approach in patients with ischemic cardiomyopathy.

LV lead implantation at sites of scar (even in "optimal" anatomic location) has been associated with poor response to CRT; whether regular use of imaging to guide lead targeting (e.g. cardiac MRI) is advantageous requires further investigation. Finally, recent work has shown that conventional "anatomically- guided" LV lead targeting is rarely associated with optimal hemodynamic improvement at the time of implant. And although the degree of acute hemodynamic improvement has been associated with greater LV reverse remodeling, whether or not this approach is superior to anatomic, electrical, or mechanical targeting strategies needs further prospective evaluation. Patients with non-LBBB do not respond to CRT as well as those with LBBB. The reason for this is probably because of the one-size-fits-all strategy and placement of the lead along the lateral wall. The electrical activation sequence in non-LBBB may be quite different from that in LBBB. Investigators have examined electrical delay and have found that accessing the area that is most electrically delayed in non-LBBB translates into a much better outcome. ${ }^{14}$ This is being evaluated prospectively in the ENHANCE-CRT study to determine the outcomes of individualized targeting in patients with non-LBBB.

Strategies to overcome potential limitations in lead targeting include use of multisite pacing, surgical implantation of epicardial leads, and endocardial pacing. The advent of Quadripolar leads represents a major improvement in implantation technology and has reduced the need for epicardial approaches. They allow us the opportunity to use a variety of different vectors to achieve better individualized activation of the LV within patients. Quadripolar leads allow us to place a stable LV lead, they help us avoid phrenic nerve pacing, and then perform non-apical pacing. Among the many procedural advantages, the quadripolar lead shaves time off of the procedure and at the same times provides the operator with a level of confidence that he or she will be able to implant the lead without encountering issues with phrenic nerve pacing. Data from the MORE CRT study ${ }^{15}$ and other studies have shown that this translates into better clinical outcomes. The results of the MORE CRT study indicate that quadripolar leads perform much better than bipolar leads. There is evidence that quadripolar leads are associated with much lower incidences of intraprocedural and immediate post-procedural adverse events, with an improvement in freedom from events, which also translates into better clinical outcomes. Some retrospective studies have examined quadripolar leads as registries and found that quadripolar leads have better clinical outcomes with reduced hospitalization for heart failure. However, prospective studies are still needed to validate these outcomes.

Quadripolar leads allow us to measure the electrical delay of different electrodes, thereby allowing us to pace the heart from the most electrically delayed electrode, giving us a much larger span of the LV myocardium that can be recruited for appropriate individualized pacing. Leads that are placed in the apical region are often associated with a poor clinical outcome. Measuring the electrical delay off of each electrode in a particular quadripolar lead and selecting the electrode that is most delayed has been shown to translate into better clinical outcomes. Kandala et al. ${ }^{16}$ studied electrical delay within apical lead locations in 45 patients and found that those patients with longer QLV had better outcomes even though the anatomical position of the lead was in the apical region.

Quadripolar leads also allow us to deliver sequential LV impulses or pulses from two or more electrodes in a sequential pattern. This can result in better activation of the LV myocardium and at the same time potentially recruit more myocardium, which in turn could result in better outcomes. Depending on how the LV lead lies across the ventricular myocardium, the sequential activation of two electrodes may vary from individual to individual and not just in a fixed conventional pattern. Studies with sequential pacing or MultiPoint ${ }^{\mathrm{TM}}$ Pacing (MPP) (St. Jude Medical, Sylmar, CA) have shown significant improvement in the acute hemodynamic 
response measured either as $\mathrm{LV} \mathrm{dP} / \mathrm{dt}_{\max }, \mathrm{LV}$ stroke volume, or LV ejection fraction. Evidence suggests that the best MultiPoint ${ }^{\mathrm{TM}}$ Pacing (MPP) (St. Jude Medical, Sylmar, CA) is better than the best conventional pacing, again indicating that there is hope in the future for nonresponders who have quadripolar leads implanted. ${ }^{17,18}$ The MORE-CRT MPP study investigators are evaluating this concept.

Given the limitations of the transvenous route, alternative pacing approaches, such as endocardial pacing, have the potential to significantly impact the field of CRT. Endocardial pacing may offer more physiological depolarization of the ventricles that extends from endocardium to epicardium in the non-pathologic ventricle. Several techniques for endocardial pacing including trans-apical, trans-aortic, and trans-septal have been proposed. Even more recently, the WiSE-CRT (Wireless Stimulation Endocardially for CRT) study demonstrated the feasibility and safety of an endocardial LV pacing system utilizing a leadless ultrasound-based algorithm. ${ }^{19}$

\section{Conclusion}

There are several determinants of response for resynchronization therapy. Patient selection is important. We are still limited by our abilities with imaging, and we are still using QRS morphology. However, several imaging strategies under development will aid in patient selection and thereby enhance response. With lead implantation, anatomical targeting is not specific enough. We need to individualize our strategy through targeting electrical delay, targeting mechanical dyssnynchrony, and avoiding scar and ischemia. The quadripolar lead gives us the opportunity to recruit and resynchronize more myocardium. Finally, sequential pacing is an evolving concept that hopefully will improve response.

\section{References}

1. Chung ES, Leon AR, Tavazzi L, et al. Results of the Predictors of Response to CRT (PROSPECT) trial. Circulation. 2008;117(20):2608-2616.

2. Ruschitzka F, Abraham WT, Singh JP, et al. Cardiac-resynchronization therapy in heart failure with a narrow QRS complex. N Engl J Med. 2013;369(15):1395-1405.

3. Poole JE, Singh JP, Birgersdotter-Green U. QRS Duration or QRS Morphology: What Really Matters in Cardiac Resynchronization Therapy? J Am Coll Cardiol. 2016;67 (9):1104-1117.

4. Sipahi I, Carrigan TP, Rowland DY, Stambler BS, Fang JC. Impact of QRS duration on clinical event reduction with cardiac resynchronization therapy: meta-analysis of randomized controlled trials. Arch Intern Med. 2011;171(16):1454-1462.

5. Sipahi I, Chou JC, Hyden M, Rowland DY, Simon DI, Fang JC. Effect of QRS morphology on clinical event reduction with cardiac resynchronization therapy: meta-analysis of randomized controlled trials. Am Heart J. 2012;163(2):260-267.

6. Upadhyay GA, Choudhry NK, Auricchio A, Ruskin J, Singh JP. Cardiac resynchronization in patients with atrial fibrillation. A meta-analysis of prospective cohort studies. Journal of the American College of Cardiology. 2008; 52(15):1239-1246.

7. Chatterjee NA, Singh JP, Szymonifka J, et al. Incremental value of cystatin $\mathrm{C}$ over conventional renal metrics for predicting clinical response and outcomes in cardiac resynchronization therapy: The BIOCRT study. Int J Cardiol. 2016;205:43-49.

8. Bose A, Kandala J, Upadhyay GA, et al. Impact of myocardial viability and left ventricular lead location on clinical outcome in cardiac resynchronization therapy recipients with ischemic cardiomyopathy. I Cardiovasc Electrophysiol. 2014;25(5):507-513.

9. Singh JP, Klein HU, Huang DT, et al. Left ventricular lead position and clinical outcome in the multicenter automatic defibrillator implantation trial-cardiac resynchronization therapy (MADIT-CRT) trial. Circulation. 2011;123(11): 1159-1166.

10. Linde C, Gold MR, Abraham WT, et al. Long-term impact of cardiac resynchronization therapy in mild heart failure: 5-year results from the REsynchronization reVErses Remodeling in Systolic left vEntricular dysfunction (REVERSE) study. Eur Heart J. 2013;34(33):2592-2599.

11. Singh JP, Fan D, Heist EK, et al. Left ventricular lead electrical delay predicts response to cardiac resynchronization therapy. Heart Rhythm. 2006;3(11):1285-1292.

12. Saba S, Marek J, Schwartzman D, et al. Echocardiographyguided left ventricular lead placement for cardiac resynchronization therapy: results Results of the Speckle Tracking Assisted Resynchronization Therapy for Electrode Region trial. Circ Heart Fail. May;6(3):427-434.

13. de Roest GJ, Wu L, de Cock CC, et al. Scar tissue-guided left ventricular lead placement for cardiac resynchronization therapy in patients with ischemic cardiomyopathy: an acute pressure-volume loop study. Am Heart J. 2014;167 (4):537-545.

14. Kandala J, Upadhyay GA, Altman RK, et al. QRS morphology, left ventricular lead location, and clinical outcome in patients receiving cardiac resynchronization therapy. Eur Heart J. 2013;34(29):2252-2262.

15. Boriani G., et al. (2014). Cardiac resynchronization therapy with a novel quadripolar electrode lead decreases complications at six months: preliminary results of the MORE-CRT. Abstract presented at European Society of Cardiology Congress (ESC) 2014; August 31, 2014; Barcelona, Spain.

16. Kandala J, Upadhyay GA, Altman RK, et al. Electrical delay in apically positioned left ventricular leads and clinical outcome after cardiac resynchronization therapy. J Cardiovasc Electrophysiol. 2013;24(2):182-187.

17. Pappone C, Ćalović Z, Vicedomini G, et al. Multipoint left ventricular pacing improves acute hemodynamic response assessed with pressure-volume loops in cardiac resynchronization therapy patients. Heart Rhythm. 2014;11(3):394-401.

18. Rinaldi CA, Leclercq C, Kranig W, et al. Improvement in acute contractility and hemodynamics with multipoint pacing via a left ventricular quadripolar pacing lead. J Interv Card Electrophysiol. 2014;40(1):75-80.

19. Auricchio A, Delnoy PP, Butter C, et al. Feasibility, safety and short-term outcome of leadless ultrasound-based endocardial left ventricular resynchronization in heart faiure patients: results of the wireless stimulation endocardially for CRT (WiSE-CRT) study. Europace. 2014;16(5):681-688. 